\title{
Age effect on bone mineral density changes in breast cancer patients receiving anastrozole: results from the ARBI prospective clinical trial
}

\author{
Christos Markopoulos • Evagelos Tzoracoleftherakis • Dimitrios Koukouras • \\ Basileios Venizelos • Vasilios Zobolas • John Misitzis • Grigorios Xepapadakis • \\ Helen Gogas
}

Received: 9 February 2012/Accepted: 10 April 2012/Published online: 3 May 2012

(c) The Author(s) 2012. This article is published with open access at Springerlink.com

\begin{abstract}
Purpose We investigated whether age at anastrozole (A) initiation influences the effect of treatment on bone mineral density (BMD). We conducted a post hoc analysis of the dataset of Arimidex Bone Mass Index Oral Bisphosphonates prospective trial, studying the effect of risedronate $(\mathrm{R})$ on $\mathrm{BMD}$ of postmenopausal, early breast cancer patients receiving A.

Methods Patients were stratified into those with normal BMD or mild osteopenia $(T>-2)$ receiving A-only and patients with mild or severe osteopenia $(T \leq-2)$ or osteoporosis $(T<-2.5)$ receiving $\mathrm{A}$ and per os $\mathrm{R}(\mathrm{A}+\mathrm{R})$. Depending on age on treatment initiation, patients were grouped into two age cohorts, above and below 65 years. BMD change in lumbar spine (LS) and hip (HP) was evaluated at 12 months. An analysis of patients with normal BMD at baseline was additionally performed.
\end{abstract}

Results Among patients receiving A-only, women $\leq 65$ years were more likely to have a decrease in LS-BMD

Trial registration: ClinicalTrials.gov Identifier NCT00809484.

C. Markopoulos · E. Tzoracoleftherakis · D. Koukouras .

B. Venizelos · V. Zobolas · J. Misitzis - G. Xepapadakis ·

H. Gogas

Hellenic Society of Breast Surgeons, 6 Eslin Street,

11523 Athens, Greece

C. Markopoulos $(\square)$

8 Iassiou Street, 11521 Athens, Greece

e-mail: cmarkop@hol.gr than older $(p=0.034)$. HP-BMD decrease at 12 months was not related to age $(p=0.182)$. In patients with mild or severe osteopenia or osteoporosis, treated with $\mathrm{A}+\mathrm{R}$, no age effect was observed for LS or HP ( $p=0.099$ and $p=0.939$, respectively). Among patients with normal BMD at baseline, the age effect on LS-BMD change was more profound ( $p=0.026$ ).

Conclusions Our study suggests that younger postmenopausal women with normal BMD or mild osteopenia receiving A-only face an increased risk of bone loss in LS. Among patients with mild or severe osteopenia or osteoporosis treated with $\mathrm{A}+\mathrm{R}, 12$ months LS or HP BMD variations were configured regardless of age group.

Keywords Breast cancer - Aromatase inhibitors ·

Anastrozole · Bisphosphonates · Risedronate ·

Bone mineral density

$\begin{array}{ll}\text { Abbreviations } \\ \text { A } & \text { Anastrozole } \\ \text { AI } & \text { Aromatase inhibitor } \\ \text { ARBI } & \text { Arimidex bone mass index and oral } \\ & \text { bisphosphonates } \\ \text { ATAC } & \text { Arimidex, tamoxifen alone or in combination } \\ \text { BMD } & \text { Bone mineral density } \\ \text { BP } & \text { Bisphosphonate } \\ \text { DEXA } & \text { Dual-energy X-ray absorptiometry } \\ \text { ECOG } & \text { Eastern cooperative oncology group } \\ \text { HP } & \text { Hip } \\ \text { LS } & \text { Lumbar spine } \\ \text { R } & \text { Risedronate } \\ \text { SD } & \text { Standard deviation } \\ \text { SERMs } & \text { Selective estrogen receptor modulators } \\ \text { T } & \text { T-score } \\ \text { WHO } & \text { World Health Organization }\end{array}$




\section{Introduction}

The principle treatment options for postmenopausal patients with hormone receptor (HR)-positive breast cancer are third-generation aromatase inhibitors (AIs) and selective estrogen receptor modulators (SERMs). Adjuvant therapy with AIs has significant benefits over SERMs in terms of disease-free survival and safety (Rugo 2008; Baum et al. 2003; Coombes et al. 2004; Goss et al. 2005; Gibson et al. 2009; Howell et al. 2005; Markopoulos 2010), and it is recommended by International Scientific Associations (Burstein et al. 2010; Carlson et al. 2006; NCCN 2011; NICE 2009).

Breast cancer and treatment increases the risk of osteoporosis, falls, and fractures in women affected (Chen et al. 2005a, b, 2009; Kanis et al. 1999). AIs reduce systemic estrogen through $98 \%$ inhibition of the aromatase enzyme (Geisler et al. 1996, 2002; Dixon et al. 2008), and this estrogen deficiency is associated with an accelerated bone loss. Bisphosphonates (BPs) are considered effective supportive therapy in the decrease in skeletal complications in different types of cancers, including breast cancer (Body et al. 1998; Aapro et al. 2008; Markopoulos et al. 2010). BPs inhibit bone resorption by interfering with osteoclast activation and by promoting osteoclast apoptosis (Body et al. 1998; Fleisch 2002; Ashcroft et al. 2003; Gnant and Eidtmann 2010). Clinical evidence supports their use as add-ons to AIs as a protective measure against osteoporosis (Gnant et al. 2007; Brufsky et al. 2009; Bundred et al. 2008; Lester et al. 2008; Van et al. 2010).

Our group recently published the results of the Arimidex Bone Mass Index and Oral Bisphosphonates (ARBI) multicenter, prospective, open-label study on the effect of the $\mathrm{BP}$ risedronate on BMD in postmenopausal, early breast cancer patients scheduled to receive anastrozole (Markopoulos et al. 2010). The ARBI study demonstrated that postmenopausal patients with normal BMD before starting anastrozole had a low risk of developing osteoporosis during the first 2 years of treatment. Furthermore, risedronate co-administration significantly increased BMD levels in patients with pre-treatment osteopenic to osteoporotic status (Markopoulos et al. 2010).

It is still not known, however, whether AIs affect bone density in the same way within different age groups of postmenopausal women. This is particularly interesting because estrogen deficiency leads to bone density decreases with age: every year, 1 of 3 women above 65 years has a fall and sustains fractures (ESHRE Capri Workshop Group 2010; Sambrook and Cooper 2006; Khosla et al. 1997), and 65 years of age is an indication for measuring BMD in osteoporosis guidelines (National Osteoporosis Foundation 2010; O'Neill et al. 2004; Papaioannou et al. 2010). Furthermore, recent research results indicate that younger patients on A treatment may face risk of increased boneresorption rates (Powell et al. 2011).

In this context, we conducted this post hoc analysis of the ARBI dataset to determine the effect of age on anastrozole-induced bone loss.

\section{Patients and methods}

Study design

The ARBI study was multicenter, prospective, open-label study on the effect of the BP risedronate on BMD in postmenopausal, early breast cancer patients scheduled to receive anastrozole (Trial registration: ClinicalTrials.gov Identifier NCT00809484).

Patients were to have undergone primary surgery and chemotherapy (if indicated). Exclusion criteria were druginduced menopause, evidence of metastatic bone disease, previous hip fractures or prostheses, bone metabolism disorders, untreated hypocalcemia, previous treatment with selective estrogen receptor modulators, hormone-replacement therapy, or bisphosphonates (BPs), and liver or renal dysfunction. All women received Anastrozole (ArimidexTM AstraZeneca, London, UK) $1 \mathrm{mg}$ /day and were followed up for 24 months. All patients had to give informed consent prior to enrollment in the study. Full local ethics committee approval was successfully obtained in all sites recruiting patients for the study, and national ethics committee approval of the trial protocol was also obtained. The design and results of the ARBI trial have been previously reported (Markopoulos et al. 2010).

\section{Patient groups}

A total of 213 postmenopausal patients with HR-positive breast cancer were enrolled into the ARBI study.

Participants were assigned to three risk groups (Fig. 1) for developing aromatase inhibitor-associated bone loss based on their baseline BMD T-score measured in lumbar spine (LS) and hip (HP):

- low risk with a normal $T$-score $\geq-1$ at both sites

- mild-to-moderate risk with $T$-score $<-1$ at either site and $>-2$ at both sites

- high risk with a $T$-score $\leq-2$ at either site.

Patients in low risk group $(n=50)$ with normal BMD at baseline ( $T$-score $\geq-1$ at both sites) received treatment with Anastrozole alone. The medium risk group $(n=70)$ was randomized to receive Risedronate in addition to Anastrozole $(n=37)$ or Anastrozole alone $(n=33)$, and Risedronate ( $35 \mathrm{mg} /$ week) was administered to all high risk patients $(n=93)$. 
Fig. 1 Schema of the post hoc age subgroup analysis. A, anastrozole; R, Risedronate; BMD, bone mineral density; DEXA, dual-energy X-ray absorptiometry; HP, hip; LS, lumbar spine

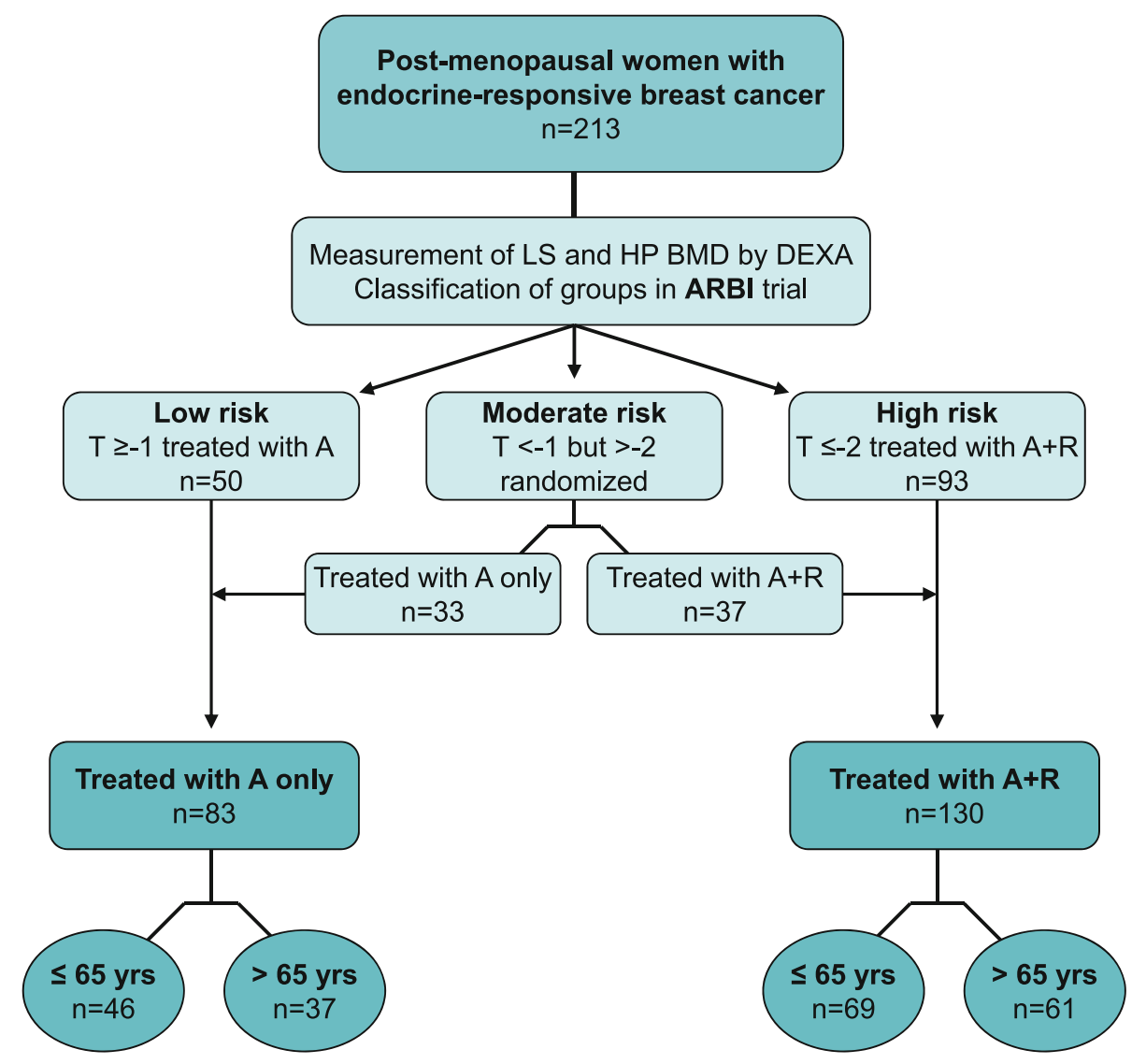

Additionally, all patients on study received daily supplements of Vitamin D (400 IU/day) and Calcium $(1,000 \mathrm{mg} / \mathrm{day})$. This is because it is known that older, postmenopausal women are at increased risk of developing vitamin $\mathrm{D}$ insufficiency because they may have inadequate intakes of vitamin D as well as of calcium, and additionally skin cannot synthesize the vitamin efficiently by aging (Institute of Medicine, Food and Nutrition Board 2010). As calcium is necessary for maintaining bone health and vitamin D is important for the absorption of calcium from the stomach, supplements were given to prevent as possible the effect that their insufficient intake would have had on BMD and influence the effect of medicines under study.

\section{Assessments}

The primary endpoint of the ARBI study was to investigate the effect of $\mathrm{R}$ in the randomized arms measured in both LS and HP at 12 months. BMD levels were evaluated at baseline before anastrozole administration, at 12, and at 24 months (secondary endpoint), by dual-energy X-ray absorptiometry (DEXA).

Patient demographic data, Eastern Cooperative Oncology Group (ECOG) Performance Status (Oken et al. 1982), and fracture history were recorded at baseline.
Formulation of the post hoc statistical analysis

The aim of this unplanned subgroup analysis was to explore whether age at baseline differentiates the effect of A on BMD change at 12 months post-treatment initiation. Depending on age on treatment initiation, patients were grouped into two age cohorts above and below 65 years, since this is the threshold for measuring BMD in osteoporosis guidelines (National Osteoporosis Foundation 2010; O'Neill et al. 2004; Papaioannou et al. 2010).

In the present analysis, we stratified the ARBI study patients (low risk treated with A-only, moderate with or without $\mathrm{R}$ and high risk with $\mathrm{R}$ ) into two groups according to the administration or not of Risedronate: (a) patients with normal BMD or mild osteopenia $(T>-2)$ receiving A-only and (b) patients with mild or severe osteopenia $(T \leq-2)$ or osteoporosis $(T<-2.5)$ receiving $\mathrm{A}$ and per os $\mathrm{R}(\mathrm{A}+\mathrm{R})$. The classification of patients as described above is presented in Fig. 1.

The outcome measure of change at 12 months posttreatment initiation was calculated as the ratio $12 \mathrm{~m} /$ baseline and expressed as percentage. Comparisons between the age groups were performed using the $t$ test. $95 \%$ confidence intervals (CI) for changes as well as for the difference between changes are reported in all cases in order to 
facilitate the assessment of the clinical significance of the findings.

Since baseline BMD levels may affect the temporal variation of BMD, we also performed a covariance analysis using baseline BMD as a confounding factor for the effect of age to account for possible predisposition bias caused by baseline imbalances on BMD (interaction effect). All statistical tests were evaluated at the $5 \%$ level of significance.

\section{Results}

Of all patients, $54.5 \%(116 / 213)$ were 65 years or younger and $45.5 \%$ (97/213) were older (Table 1). The ECOG status was 0 for nearly all $\leq 65$-year-old patients ( $\%$ range: 97.9-100.0) and for most $>65$-year-old patients (\% range: 75.0-77.0). Seven patients had sustained traumatic fractures 3-56 years before study enrollment, none of the LS or HP.

Figure 2a shows the average percent BMD change from baseline and the $95 \% \mathrm{CI}$ for LS and HP in patients with normal BMD or mild osteopenia at baseline receiving treatment with A-only. Mean BMD percent change in LS was $-5.8 \%(95 \%$ CI: $-9.5 \%,-2.1 \%)$ for patients $\leq 65$ and $-0.5 \%$ (95\% CI: $-3.7 \%, 2.6 \%$ ) for patients $>65$. The difference in density loss of $5.3 \%$ (95\% CI: $0.4 \%$, $10.1 \%$ ) between the age groups was statistically significant $(p=0.034$, Table 1). Mean BMD percent change in HP was $-1.4 \%(95 \% \mathrm{CI}:-5.9 \%, 3.0 \%)$ for patients $\leq 65$ and $-5.3 \%(95 \% \mathrm{CI}:-8.5 \%,-2.2 \%)$ for patients $>65$. The difference in density loss of $3.9 \%$ (95\% CI: $-1.9 \%$, $9.7 \%$ ) between the age groups was not statistically significant $(p=0.182$, Table 1$)$.

In patients with mild or severe osteopenia or osteoporosis receiving $\mathrm{A}+\mathrm{R}$ (Fig. 2B), mean $\mathrm{BMD}$ percent change in LS was $+4.2 \%$ (95\% CI: $1.5 \%, 6.9 \%$ ) for patients $\leq 65$ and $+8.1 \%$ (95\% CI: $4.0 \%, 12.2 \%$ ) for patients $>65$. The difference in density gain of $3.9 \%$ (95 \% CI: $-0.7 \%, 8.5 \%$,) between the age groups was not statistically significant $(p=0.099$, Table 1$)$. Mean BMD percent change in HP was $-0.2 \%$ (95\% CI: $-3.4 \%$, $2.9 \%)$ for patients $\leq 65$ and $-0.4 \%(95 \% \mathrm{CI}:-3.9 \%$, $3.1 \%$ ) for patients $>65$. The difference in density loss of $0.2 \%$ (95\% CI: $-4.5 \%, 4.9 \%$ ) between the age groups was not statistically significant $(p=0.939$, Table 1$)$.

Figure 3 shows the average percent BMD change from baseline and the $95 \% \mathrm{CI}$ for LS and HP for the substratum of 50 patients with normal BMD at baseline. Mean BMD percent change in LS was $-9.1 \%(95 \% \mathrm{CI}:-13.2 \%$, $-5.1 \%$ ) for patients $\leq 65$ and $-2.6 \%$ (95\% CI: $-6.6 \%$, $1.3 \%$ ) for patients $>65$. The difference in density loss of $6.5 \%$ (95\% CI: $0.8 \%, 12.2 \%$ ) between the age groups was statistically significant $(p=0.026$, Table 2$)$. Mean

Table 1 Patient baseline characteristics by age group and treatment strata

\begin{tabular}{|c|c|c|c|c|c|c|}
\hline & \multicolumn{2}{|l|}{ A } & \multicolumn{2}{|l|}{$A+R$} & \multicolumn{2}{|l|}{ Total } \\
\hline & $\leq 65$ & $>65$ & $\leq 65$ & $>65$ & $\leq 65$ & $>65$ \\
\hline \multicolumn{7}{|l|}{ Age (years) } \\
\hline $\mathrm{N}$ & 47 & 36 & 69 & 61 & 116 & 97 \\
\hline Mean \pm SD & $57 \pm 4.7$ & $71 \pm 4.6$ & $58 \pm 4.1$ & $72 \pm 4.5$ & $58 \pm 4.4$ & $72 \pm 4.6$ \\
\hline \multicolumn{7}{|l|}{ BMD LS } \\
\hline Mean \pm SD & $1.04 \pm 0.12$ & $1.02 \pm 0.14$ & $0.84 \pm 0.14$ & $0.80 \pm 0.16$ & $0.91 \pm 0.16$ & $0.88 \pm 0.19$ \\
\hline \multicolumn{7}{|l|}{ BMD HP } \\
\hline Mean \pm SD & $0.88 \pm 0.13$ & $0.84 \pm 0.10$ & $0.76 \pm 0.09$ & $0.71 \pm 0.11$ & $0.81 \pm 0.12$ & $0.76 \pm 0.12$ \\
\hline \multicolumn{7}{|l|}{ BMI } \\
\hline \multirow[t]{2}{*}{ Mean \pm SD } & $28.76 \pm 5.51$ & $29.57 \pm 3.85$ & $26.89 \pm 5.02$ & $28.62 \pm 4.22$ & $27.65 \pm 5.28$ & $28.97 \pm 4.09$ \\
\hline & $\mathrm{N}(\%)$ & & & & & \\
\hline \multicolumn{7}{|l|}{ ECOG status } \\
\hline 0 & $46(97.9)$ & $27(75.0)$ & $69(100.0)$ & $47(77.0)$ & $115(99.1)$ & $74(76.3)$ \\
\hline 1 & $1(2.1)$ & $9(25.0)$ & $0(0.0)$ & $14(23.0)$ & $1(0.9)$ & $23(23.7)$ \\
\hline \multicolumn{7}{|l|}{ Fracture history } \\
\hline No & $41(87.2)$ & 35 (97.2) & $67(97.1)$ & $54(88.5)$ & $108(93.1)$ & 89 (91.8) \\
\hline $\mathrm{Yes}^{\mathrm{a}}$ & $3(6.4)$ & $0(0.0)$ & $1(1.4)$ & $3(4.9)$ & $4(3.4)$ & $3(3.1)$ \\
\hline Not reported & $3(6.4)$ & $1(2.8)$ & $1(1.4)$ & $4(6.6)$ & $4(3.4)$ & $5(5.2)$ \\
\hline
\end{tabular}

$A$ anastrozole, $R$ risedronate, $B M D$ bone mineral density, $B M I$ body mass index, ECOG eastern cooperative oncology group, $S D$ standard deviation

${ }^{a}$ Traumatic fractures only; between 3 and 56 years before enrollment in the study; none in the hip (HP) or lumbar spine (LS) 

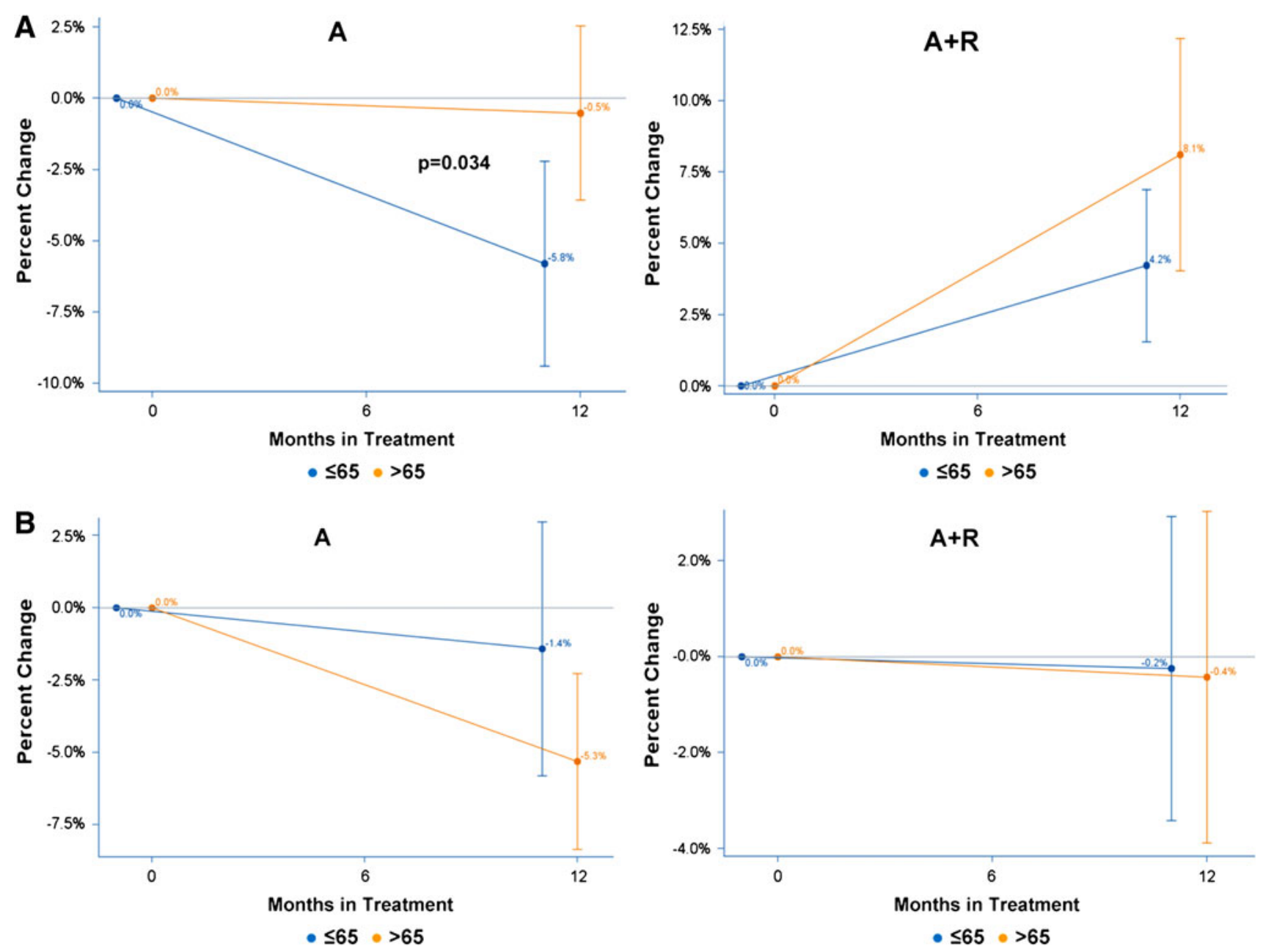

Fig. 2 a Average BMD change from baseline at lumbar spine $(L S)$ by age group, in patients on Anastrozole-only (A) and on Anastrozole plus Risedronate $(\mathrm{A}+\mathrm{R})$. b Average BMD change from baseline at

BMD percent change in HP was $-3.8 \%$ (95 \% CI: $-7.4 \%,-0.2 \%)$ for patients $\leq 65$ and $-3.9 \%$ (95\% CI: $-6.8 \%,-1.1 \%)$ for patients $>65$. The difference in density loss of $0.1 \%$ (95\% CI: $-4.7 \%, 5.0 \%$ ) between the age groups was not statistically significant $(p=0.957$, Table 2).

Covariance analysis examined whether the effect of age is truly caused by differences in baseline BMD (Table 3 ). The age effect on LS changes at 12 months in patients with normal BMD or mild osteopenia at baseline remained statistically significant even after adjustment by baseline BMD values $(p=0.0295)$. Moreover, the estimate of the adjusted difference was almost identical to the unadjusted $(-5.147$ and $-5.3 \%$, respectively). The negative effect of baseline BMD value on LS changes at 12 months is statistically significant in both treatment groups (A and $\mathrm{A}+\mathrm{R}, p$ value $=0.0098$ and $p$ value $<0.001$, respectively). This means that patients with higher baseline BMD levels are more likely to present larger loss or smaller

hip (HP) by age group, in patients receiving Anastrozole-only (A) or Anastrozole plus Risedronate $(\mathrm{A}+\mathrm{R})$

increase at 12 months compared with patients with smaller baseline BMD levels. However, regarding HP, no statistically significant associations were detected (Table 3; results are shown without the interaction effect that was not significant).

\section{Discussion}

We conducted this post hoc analysis of the ARBI dataset to evaluate the difference in BDM changes between age groups. Our results indicate that women $>65$ years are more likely to experience larger increases or smaller decreases in their LS-BMD levels than women $\leq 65$ years. In HP, no statistically significantly differences were recorded between age groups.

In patients with normal BMD or mild osteopenia at baseline receiving treatment with A-only, our observation of a systematic deterioration in BMD levels in patients 

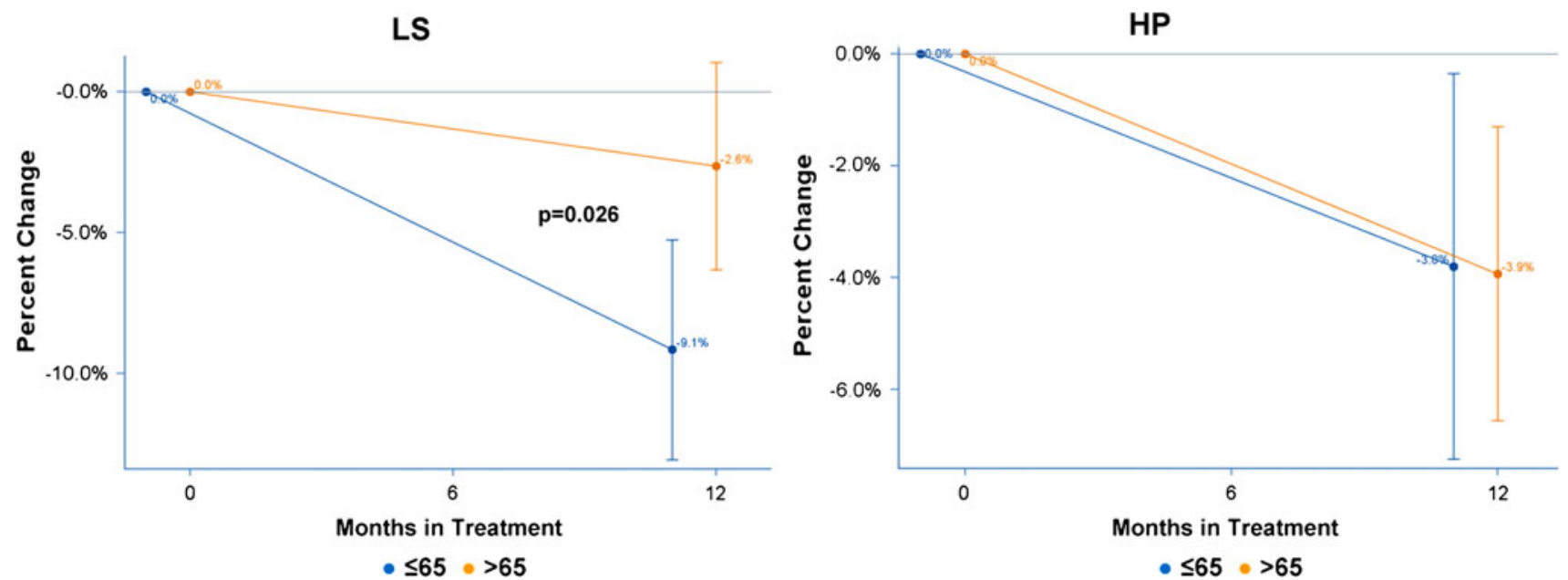

Fig. 3 Average BMD change at lumbar spine $(L S)$ and hip $(H P)$ by age group, in 50 patients with normal BMD at baseline, receiving A-only

Table 2 Average BMD change from baseline (95\% CI) by age group at 12 months

\begin{tabular}{llll}
\hline & $\leq 65$ & $>65$ & Difference \\
\hline Based on treatment & & & \\
LS & $-5.8 \%(-9.5 \%,-2.1 \%)$ & $-0.5 \%(-3.7 \%, 2.6 \%)$ & $5.3 \%(0.4 \%, 10.1 \%)$ \\
A & $4.2 \%(1.5 \%, 6.9 \%)$ & $8.1 \%(4.0 \%, 12.2 \%)$ & $3.9 \%(-0.7 \%, 8.5 \%)$ \\
A $+\mathrm{R}$ & & $-5.3 \%(-8.5 \%,-2.2 \%)$ & $3.9 \%(-1.9 \%, 9.7 \%)$ \\
HP & $-1.4 \%(-5.9 \%, 3.0 \%)$ & $-0.4 \%(-3.9 \%, 3.1 \%)$ & $0.2 \%(-4.5 \%, 4.9 \%)$ \\
A & $-0.2 \%(-3.4 \%, 2.9 \%)$ & 0.034 \\
A $+\mathrm{R}$ & $-9.1 \%(-13.2 \%,-5.1 \%)^{\mathrm{a}}$ & $-2.6 \%(-6.6 \%, 1.3 \%)^{\mathrm{b}}$ & 0.182 \\
Patients $(N=50)$ & with normal BMD at baseline, receiving A alone & 0.939 \\
LS & $-3.8 \%(-7.4 \%,-0.2 \%)^{\mathrm{c}}$ & $-3.9 \%(-6.8 \%,-1.1 \%)^{\mathrm{d}}$ & $0.1 \%(-4.7 \%, 5.0 \%)$ \\
HP
\end{tabular}

$L S$ lumbar spine, $H P$ hip, $A$ anastrozole, $R$ risedronate, $B M D$ bone mineral density, $C I$ confidence interval

${ }^{\mathrm{a}} N=20 ;{ }^{\mathrm{b}} N=14 ;{ }^{\mathrm{c}} N=21 ;{ }^{\mathrm{d}} N=14$

Table 3 Covariance analysis, age effect on the percent change from baseline adjusted by baseline BMD values

\begin{tabular}{llllll}
\hline Effect & LS & & & HP & \\
\cline { 2 - 3 } & Estimate & $p$ value & & Estimate & $p$ value \\
\hline A & & & & \\
Age $(\leq 65$ vs. $>65)$ & -0.05147 & 0.0295 & 0.03734 & 0.1996 \\
Baseline BMD & -0.2551 & 0.0098 & -0.2029 & 0.0997 \\
A $+\mathrm{R}$ & & & & \\
$\quad$ Age $(\leq 65$ vs. $>65)$ & 0.02446 & 0.2649 & -0.00425 & 0.8595 \\
Baseline BMD & -0.2917 & $<0.0001$ & -0.05986 & 0.4432 \\
\hline
\end{tabular}

$L S$ lumbar spine, $H P$ hip, $A$ anastrozole, $R$ risedronate, $B M D$ bone mineral density

$\leq 65$ years could be possibly attributed to greater skeletal changes occurring in younger patients when receiving anastrozole. This greater change in BMD when anastrozole is administered to younger women was shown by Powell et al. 2011. They compared the levels of the bone-resorption marker uNTx (urinary cross-linked N-telopeptide of type I collagen) in newly diagnosed women with breast cancer who were receiving anastrozole and were above or below the age of 70 years with that of healthy women of the same age group ( $<70$ or $\geq 70$ years). The group of younger women had statistically significantly higher levels of the bone-resorption marker compared with their healthy counterparts, while the older age group had similar levels compared with healthy women. The uNTx levels in younger women on anastrozole were similar to those in elderly women, both healthy and on anastrozole. In younger women, uNTx exceeded normal levels but not in older women. This could be attributed to higher levels of free estradiol in younger, postmenopausal women, which allows for more marked effects with the aromatase inhibitors (Powell et al. 2011). Supporting data from the literature show that there is a decline in free estradiol levels 
with aging (Bjornerem et al. 2004); this seems to be caused by a rise of sex hormone globulin levels found in elderly women rather than due to decreased levels of total estradiol in this age group (Sharp et al. 1996; Laughlin et al. 2000). However, differences in the degree of BMD change caused by anastrozole between elderly breast cancer patients and their younger counterparts could also be due to some extend to a different sensitivity of hormone receptors to circulating estradiol, developed by aging. This hypothesis needs further investigation.

Comparing the anastrozole effect on LS and HP, in the ATAC (Eastell et al. 2006) as well as in the SABRE trial (Van Poznak et al. 2010), anastrozole significantly reduced BMD of both LS and HP; notably, in both trials, patients showed a greater BMD loss in the lumbar region (LS) than at femoral sites (HP). In our study, results are in the same direction with the above trials, although changes in HP were not statistically significant in our study population; anastrozole had a negative effect on femoral BMD as well, whereas risedronate was shown that can prevent this BMD loss (Table 2; Fig. 2a). This difference in the degree of BMD change according to sites (LS and HP) could be attributed to different sensitivity of receptors to AI-induced estrogen deprivation of lumbar region and femoral sites with advancing age.

Crivellari et al. 2008 investigated differences in response to letrozole treatment and adverse events by age groups but did not assess bone marker profiles, only bone fractures, which were similar across different age groups. The ATAC trial (Arimidex Tamoxifen Alone or in Combination) showed greater BMD losses in anastrozoletreated women who experienced menopause within the last 4 years than in those who were more than 4 years postmenopausal (Eastell et al. 2006). A subsequent analysis of the same study for the investigation of potential risk factors for joint symptoms showed no detectable effect of age (Sestak et al. 2008).

Our literature search did not retrieve any other studies comparing the impact of AIs on bone marker profile measurements depending on the age of the patient. Our results and those published by Powell et al. 2011 indicate the need for age group comparison of bone markers and further evaluation of the impact the AIs have on different age groups of patients. AIs have been found to have higher efficacy than Tamoxifen (Rugo 2008; Baum et al. 2003; Coombes et al. 2004; Goss et al. 2005; Gibson et al. 2009; Howell et al. 2005; Markopoulos 2010), and there is a general recommendation for the administration of an $\mathrm{AI}$ at some point during the adjuvant hormonal treatment of postmenopausal patients with hormone receptor positive early breast cancer (Burstein et al. 2010). However, there is concern about the negative effect they have on BMD in contrast to Tamoxifen and this is important for both, younger postmenopausal women losing bone mass due to recent menopause and aging, and elderly women often having already osteopenia-osteoporosis but different life expectancy. Therefore, it is very important to explore possible differences in the effect that AIs might have on BMD according to age of patients and to BMD status before the administration of an $\mathrm{AI}$, so appropriate supportive measures could be taken in clinical practice.

Moreover, our findings render the general perception to feel "safe" considering "bone loss" when starting adjuvant treatment with an AI in patients having normal BMD before treatment, questionable. Maybe young, postmenopausal patients starting AI treatment should be followed for BMD changes, especially in LS, despite normal BMD at baseline.

Notably, our results are limited by the fact that this is an unplanned subgroup analysis of the ARBI trial, and they were not powered to investigate such hypothesis. Despite this, they do indicate that a future study aiming to explicitly address this specific issue of age-related BMD changes in postmenopausal breast cancer patients on adjuvant treatment with AIs is justified.

In conclusion, our results suggest that among patients with normal BMD or mild osteopenia receiving A-only, younger women face an increased risk of BMD loss in LS 12 months post-treatment initiation, especially if they present with normal BMD. Among patients with mild or severe osteopenia or osteoporosis, AI treatment side effect on BMD is not related to age group.

Acknowledgments The ARBI trial and the present analysis was supported by an independent investigator research grant from AstraZeneca, which, however, had no role in the design of the study, in the collection, analysis, and interpretation of data, in the writing of the manuscript, or in the decision to submit the manuscript for publication.

Conflict of interest $\mathrm{CM}$ has received educational grants and lecture honoraria from AstraZeneca, Novartis (Basel, Switzerland), and Pfizer Inc (New York, NY, USA). ET, BV, GX, VZ, JM, DK, and HG have received unrestricted educational grants from AstraZeneca, Novartis, and Pfizer Inc.

Open Access This article is distributed under the terms of the Creative Commons Attribution License which permits any use, distribution, and reproduction in any medium, provided the original author(s) and the source are credited.

\section{References}

Aapro M, Abrahamsson PA, Body JJ et al (2008) Guidance on the use of bisphosphonates in solid tumours: recommendations of an international expert panel. Ann Oncol 19:420-432

Ashcroft AJ, Davies FE, Morgan GJ (2003) Aetiology of bone disease and the role of bisphosphonates in multiple myeloma. Lancet Oncol 4:284-292 
Baum M, Buzdar A, Cuzick J et al (2003) Anastrozole alone or in combination with tamoxifen versus tamoxifen alone for adjuvant treatment of postmenopausal women with early-stage breast cancer: results of the ATAC (Arimidex, Tamoxifen Alone or in Combination) trial efficacy and safety update analyses. Cancer 98:1802-1810

Bjornerem A, Straume B, Midtby M et al (2004) Endogenous sex hormones in relation to age, sex, lifestyle factors, and chronic diseases in a general population: the Tromso study. J Clin Endocrinol Metab 89:6039-6047

Body JJ, Bartl R, Burckhardt P et al (1998) Current use of bisphosphonates in oncology. International Bone and Cancer Study Group. J Clin Oncol 16:3890-3899

Brufsky AM, Bosserman LD, Caradonna RR et al (2009) Zoledronic acid effectively prevents aromatase inhibitor-associated bone loss in postmenopausal women with early breast cancer receiving adjuvant letrozole: Z-FAST study 36-month follow-up results. Clin Breast Cancer 9:77-85

Bundred NJ, Campbell ID, Davidson N et al (2008) Effective inhibition of aromatase inhibitor-associated bone loss by zoledronic acid in postmenopausal women with early breast cancer receiving adjuvant letrozole: ZO-FAST Study results. Cancer 112:1001-1010

Burstein HJ, Prestrud AA, Seidenfeld J et al (2010) American Society of Clinical Oncology clinical practice guideline: update on adjuvant endocrine therapy for women with hormone receptorpositive breast cancer. J Clin Oncol 28:3784-3796

Carlson RW, Hudis CA, Pritchard KI (2006) Adjuvant endocrine therapy in hormone receptor-positive postmenopausal breast cancer: evolution of NCCN, ASCO, and St Gallen recommendations. J Natl Compr Canc Netw 4:971-979

Chen Z, Maricic M, Bassford TL et al (2005a) Fracture risk among breast cancer survivors: results from the Women's Health Initiative Observational Study. Arch Intern Med 165:552-558

Chen Z, Maricic M, Pettinger M et al (2005b) Osteoporosis and rate of bone loss among postmenopausal survivors of breast cancer. Cancer 104:1520-1530

Chen Z, Maricic M, Aragaki AK et al (2009) Fracture risk increases after diagnosis of breast or other cancers in postmenopausal women: results from the Women's Health Initiative. Osteoporos Int 20:527-536

Coombes RC, Hall E, Gibson LJ et al (2004) A randomized trial of exemestane after two to three years of tamoxifen therapy in postmenopausal women with primary breast cancer. N Engl J Med 350:1081-1092

Crivellari D, Sun Z, Coates AS et al (2008) Letrozole compared with tamoxifen for elderly patients with endocrine-responsive early breast cancer: the BIG 1-98 trial. J Clin Oncol 26:1972-1979

Dixon JM, Renshaw L, Young O et al (2008) Letrozole suppresses plasma estradiol and estrone sulphate more completely than anastrozole in postmenopausal women with breast cancer. J Clin Oncol 26:1671-1676

Eastell R, Hannon RA, Cuzick J, Dowsett M, Clack G, Adams JE (2006) Effect of an aromatase inhibitor on bmd and bone turnover markers: 2-year results of the Anastrozole, Tamoxifen, Alone or in Combination (ATAC) trial (18233230). J Bone Miner Res 21:1215-1223

ESHRE Capri Workshop Group (2010) Bone fractures after menopause. Hum Reprod Update 16:761-773

Fleisch H (2002) Development of bisphosphonates. Breast Cancer Res 4:30-34

Geisler J, King N, Dowsett M et al (1996) Influence of anastrozole (Arimidex), a selective, non-steroidal aromatase inhibitor, on in vivo aromatisation and plasma oestrogen levels in postmenopausal women with breast cancer. Br J Cancer 74:1286-1291

Geisler J, Haynes B, Anker G, Dowsett M, Lonning PE (2002) Influence of letrozole and anastrozole on total body aromatization and plasma estrogen levels in postmenopausal breast cancer patients evaluated in a randomized, cross-over study. J Clin Oncol 20:751-757

Gibson L, Lawrence D, Dawson C, Bliss J (2009) Aromatase inhibitors for treatment of advanced breast cancer in postmenopausal women. Cochrane Database Syst Rev, p CD003370

Gnant M, Eidtmann H (2010) The anti-tumor effect of bisphosphonates ABCSG-12, ZO-FAST and more.... Crit Rev Oncol Hematol 74(Suppl 1):S2-S6

Gnant MF, Mlineritsch B, Luschin-Ebengreuth G et al (2007) Zoledronic acid prevents cancer treatment-induced bone loss in premenopausal women receiving adjuvant endocrine therapy for hormone-responsive breast cancer: a report from the Austrian Breast and Colorectal Cancer Study Group. J Clin Oncol 25: $820-828$

Goss PE, Ingle JN, Martino S et al (2005) Randomized trial of letrozole following tamoxifen as extended adjuvant therapy in receptor-positive breast cancer: updated findings from NCIC CTG MA.17. J Natl Cancer Inst 97:1262-1271

Howell A, Cuzick J, Baum M et al (2005) Results of the ATAC (Arimidex, Tamoxifen, Alone or in Combination) trial after completion of 5 years' adjuvant treatment for breast cancer. Lancet 365:60-62

Institute of Medicine, Food and Nutrition Board (2010) Dietary reference intakes for Calcium and Vitamin D. National Academy Press, Washington, DC

Kanis JA, McCloskey EV, Powles T, Paterson AH, Ashley S, Spector $\mathrm{T}$ (1999) A high incidence of vertebral fracture in women with breast cancer. Br J Cancer 79:1179-1181

Khosla S, Atkinson EJ, Melton LJ III, Riggs BL (1997) Effects of age and estrogen status on serum parathyroid hormone levels and biochemical markers of bone turnover in women: a populationbased study. J Clin Endocrinol Metab 82:1522-1527

Laughlin GA, Barrett-Connor E, Kritz-Silverstein D, von Muhlen D (2000) Hysterectomy, oophrectomy and endogenous hormone levels in older women: the Rancho Bernardo study. J Clin Endocrinol Metab 85:645-651

Lester JE, Dodwell D, Purohit OP et al (2008) Prevention of anastrozole-induced bone loss with monthly oral ibandronate during adjuvant aromatase inhibitor therapy for breast cancer. Clin Cancer Res 14:6336-6342

Markopoulos C (2010) Minimizing early relapse and maximizing treatment outcomes in hormone-sensitive postmenopausal breast cancer: efficacy review of AI trials. Cancer Metastasis Rev 29:581-594

Markopoulos C, Tzoracoleftherakis E, Polychronis A et al (2010) Management of anastrozole-induced bone loss in breast cancer patients with oral risedronate: results from the ARBI prospective clinical trial. Breast Cancer Res 12:R24

National Comprehensive Cancer Network (NCCN) (2011) Clinical Practice Guideline Guidelines in Oncology: Breast Cancer, V.2.2011, Ref Type: Serial (Book, Monograph)

National Institute for Health and Clinical Excellence (NICE) (2009) Early and locally advanced breast cancer: diagnosis and treatment: full guideline

National Osteoporosis Foundation (2010) Clinician's guide to prevention and treatment of osteoporosis. National Osteoporosis Foundation, Washington, DC

Oken MM, Creech RH, Tormey DC et al (1982) Toxicity and response criteria of the Eastern Cooperative Oncology Group. Am J Clin Oncol 5:649-655

O'Neill S, MacLennan A, Bass S et al (2004) Guidelines for the management of postmenopausal osteoporosis for GPs. Aust Fam Physician 33:910-919

Papaioannou A, Morin S, Cheung AM et al (2010) Scientific Advisory Council of Osteoporosis Canada. 2010 clinical practice 
guidelines for the diagnosis and management of osteoporosis in Canada: summary. Can Med Assoc J 182:1864-1873

Powell DE, Cochrane RA, Davie MW (2011) Does anastrozole affect bone reabsorption similarly in early and late postmenopausal women? Calcif Tissue Int 88:223-230

Rugo HS (2008) The breast cancer continuum in hormone-receptorpositive breast cancer in postmenopausal women: evolving management options focusing on aromatase inhibitors. Ann Oncol 19:16-27

Sambrook P, Cooper C (2006) Osteoporosis. Lancet 367:2010-2018
Sestak I, Cuzick J, Sapunar F et al (2008) Risk factors for joint symptoms in patients enrolled in the ATAC trial: a retrospective, exploratory analysis. Lancet Oncol 9:866-872

Sharp CA, Evans SF, Ristelli L, Worsfold M, Davie MWJ (1996) Effects of low- and conventional-dose transcutaneous HRT over 2 years on bone metabolism in younger and older postmenopausal women. Eur J Clin Invest 26:763-771

Van Poznak PC, Hannon RA, Mackey JR et al (2010) Prevention of aromatase inhibitor-induced bone loss using risedronate: the SABRE trial. J Clin Oncol 28:967-975 